\title{
Novel Dihydroartemisinin Derivative Mito-DHA5 Induces Apoptosis Associated with Mitochondrial Pathway in Bladder Cancer Cells
}

\section{Linfan Xiao}

Hunan Normal University

\section{Cangcang $\mathrm{Xu}$}

Hunan Normal University

Peiyu Lin

Hunan Normal University

\section{Lingli Mu}

Hunan Normal University

Xiaoping Yang ( $\nabla$ Xiaoping.Yang@hunnu.edu.cn )

Hunan Normal University

\section{Research Article}

Keywords: Dihydroartemisinin derivative, ROS, MMP, Apoptosis, Bladder cancer

Posted Date: September 28th, 2021

DOl: https://doi.org/10.21203/rs.3.rs-757247/v1

License: (9) This work is licensed under a Creative Commons Attribution 4.0 International License. Read Full License

Version of Record: A version of this preprint was published at BMC Pharmacology and Toxicology on January 20th, 2022. See the published version at https://doi.org/10.1186/s40360-021-00542-6. 


\section{Novel dihydroartemisinin derivative Mito-DHA 5 induces apoptosis associated with mitochondrial pathway in bladder cancer cells \\ Linfan Xiao, Cangcang Xu*, Peiyu Lin, Lingli Mu*, Xiaoping Yang*}

Key Laboratory of Study and Discovery of Small Targeted Molecules of Hunan Province, Department of Pharmacy, School of Medicine, Hunan Normal University, Changsha, Hunan, China

* Corresponding authors.

Key Laboratory of Study and Discovery of Small Targeted Molecules of Hunan Province, Department of Pharmacy, School of Medicine, Hunan Normal University, Changsha, Hunan, China.

E-mail: xucangcang@hunnu.edu.cn; moulingli@sina.com; Xiaoping.Yang@hunnu.edu.cn 


\begin{abstract}
Background: Bladder cancer is the second most common genitourinary malignancy and the eleventh most common cancer worldwide. Dihydroartemisinin (DHA), a first-line antimalarial drug, has been found to have potent antitumor activity. In our previous study, a novel dihydroartemisinin derivative Mito-DHA 5 synthesized in our laboratory has a stronger anti-tumor activity than DHA. In this study, we investigated the apoptotic effect of Mito-DHA 5 on bladder cancer T24 cells and molecular mechanisms underlying.
\end{abstract}

Methods: Antitumor activity in vitro was evaluated by MTT and cloning formation assays. Mitochondrial membrane potential (MMP) was detected by JC-1 probe and ROS levels were measured by specific kit. The expression of caspase-3, Bcl-2 and Bax in T24 cells was evaluated by Western blotting.

Results: The results showed that Mito- $\mathrm{DHA}_{5}$ reduced cell viability with an $\mathrm{IC}_{50}$ value of $3.2 \mu \mathrm{M}$ in a dose-dependent manner, induced T24 cell apoptosis at both early and late stages, increased the production of ROS and decreased MMP. Mito-DHA 5 could down-regulate the expression of Bcl-2 and Caspase-3, and up-regulate the expression of Bax and cleaved Caspase-3.

Conclusion: These data suggested that Mito-DHA 5 had a potent inhibitory effect on T24 bladder cancer cell growth and induced these cells apoptosis associated with mitochondrial pathway.

Keywords: Dihydroartemisinin derivative, ROS, MMP, Apoptosis, Bladder cancer. 


\section{Background}

Bladder cancer is the second most common genitourinary malignancy and the eleventh most common cancer worldwide [1]. According to the global cancer data, there are 500000 new bladder cancer cases and 200000 deaths in the world every year [2]. Generally, transurethral resection has served as the standard treatment. However, recurrence and metastasis are often seen in clinic after this surgery [3]. Thus, intravesical chemotherapy or immunosuppressive agents are applied to treat these patients to prevent these severe events in the commonest way [4]. However, these agents have considerable side effects such as bone marrow suppression and allergic reactions [5]. Therefore, there is an urgent need to develop anticancer agents with high efficacy and low toxicity to treat bladder carcinoma.

DHA is an active metabolite of artemisinin which is widely used to treat malaria in clinic [6]. Recent years, increasing number of studies reported that DHA exhibited anti-cancer activities in different kind of cancers, such as ovarian cancer [7], lung cancer [8], esophageal cancer [9], prostate cancer [10] and colon cancer [11], etc. In contrast, chemical modification of DHA is becoming a notably research area to find novel small molecules to convey cancers. In our previous study, we found that Mito-DHA5 (Fig. 1A), which is a mitochondria-targeted derivative of DHA has more potent anti-tumor activity than DHA [12]. However, the mechanisms of action of Mito-DHA 5 remain unknown.

Mitochondria are important bioenergy factories for normal cell function and human health. In tumor cells, mitochondria are dysfunctional and cannot release apoptosis signals in time, leading to indefinite proliferation and apoptotic resistance [13]. The mitochondrial apoptosis pathway takes mitochondrial depolarization as a starting point, which is regulated by members of the Bcl-2 protein family, following the release of apoptosis signal, then activates caspase-3 to trigger apoptosis [14]. Interestingly, Farhad Poupel et al. found that DHA could induce apoptosis through mitochondrial signaling pathway in bladder cancer [15]. In order to clarify the potential mechanisms of action of Mito-DHA 5 , the effect of Mito-DHA 5 on T24, one 
of representative bladder cancer cell lines, was investigated in this study.

\section{Materials and Methods}

DHA was purchased from Energy Chemical Reagent Company (Shanghai, China). Mito-DHA 5 was synthesized in our laboratory. Compounds were dissolved in Dimethyl sulfoxide (DMSO). During the experiment the concentration of DMSO did not exceed 0.1\%. Dulbecco's modified Eagle's medium (DMEM, U.S.), fetal bovine serum (Hyclone, Logan, UT, USA), penicillin-streptomycin solution, 0.25\% trypsin and phosphate buffer (Hyclone, USA) were bought from Hyclone company. Mitochondrial Membrane Potential Detection Kit was bought from Solarbio company (Beijing, China). AnnexinV- FITC/PI Apoptosis Detection Kit was purchased from Vazyme (Nanjing, China). ROS Detection Kit, Hochest33258 Kit and Bax (AF0057)

protein antibodies were all from Beyotime company (Shanghai, China). Bcl-2 (CAS7511) protein antibody is from Bioworld. Caspase-3 (\#9662) antibody was purchased from Cell Signaling Technology company.

\section{Cell culture}

Human bladder cancer cell T24 was obtained from Dr. P Guo (Xi'an Jiaotong University). The cells were grew in Dulbecco's modified Eagle's medium (DMEM) (HyClone) contained with 10\% of FBS (HyClone) and 1\% of penicillin-streptomycin at $37^{\circ} \mathrm{C}$, in a constant temperature incubator containing $5 \%$ of $\mathrm{CO}_{2}$.

\section{MTT assay}

T24 cells were planted in 96 well plates $\left(8.0 \times 10^{3}\right.$ per well $)$ and added different concentrations $(0,3,10,30$ and $100 \mu \mathrm{M})$ of Mito-DHAs or DHA after sticking to the wall in the DMEM for 24,48 or $72 \mathrm{~h}$. Then MTT ( Solarbio $2 \mathrm{mg} / \mathrm{ml}, 50 \mu \mathrm{L}$ ) was mixed in the medium. Then removing the medium after $4 \mathrm{~h}$ incubation at $37^{\circ} \mathrm{C}$ and mixing in DMSO $(150 \mu \mathrm{L})$, then measuring OD values at $490 \mathrm{~nm}$ thourgh a microplate reader (Biotek).

\section{Clonogenic assay}

In brief, seeding $3 \times 10^{3}$ cells per well in 24 well plates. After $12 \mathrm{~h}$, using different concentrations $(0,1,2,4 \mu \mathrm{M})$ of Mito-DHA 5 or DHA treated the cells for about 7 days. Fixing T24 cells with $10 \%$ formaldehyde and then adding $0.1 \%$ crystal violet to 
stain cells. Then measuring the OD values through area scanning at $550 \mathrm{~nm}$ by using microplate reader.

\section{Hoechst 33258 staining}

Plating T24 cells in 24 well plates $\left(2.0 \times 10^{5}\right.$ cells per well) and after overnight incubating in the presence of Mito-DHA 5 at $30 \mu \mathrm{M}$ or DHA at $30 \mu \mathrm{M}$ for $48 \mathrm{~h}$ or pretreated by $5 \mathrm{mM}$ NAC (Beyotime, China) for $2 \mathrm{~h}$ then incubated in presence of Mito-DHA $5(0,10 \mu \mathrm{M})$ at various concentrations for $48 \mathrm{~h}$ to induce T24 cell apoptosis. Then T24 cells were washed three times with PBS, stained with Hoechst 33258 according to instructions. After this processing, observed by fluorescence microscope (DFC450C; Leica, Wetzlar, Germany).

\section{Cell apoptosis determination}

In brief, T24 cells $\left(5 \times 10^{5}\right.$ cells per well) were plated in 6 -well plates. After $12 \mathrm{~h}$, treating T24 cells with Mito-DHA $5(0,3,10,30 \mu \mathrm{M})$ for $48 \mathrm{~h}$. Then washing cells use cold PBS for three times and resuspending the cells in binding buffer. Adding annexin V-FITC and PI solution according to the instructions. Incubating at room temperature for 10 minutes in the dark, and then testing the sample with a flow cytometry (Becton Dickinson).

\section{Measurement of Intracellular ROS Generation}

Planting T24 cells in 12-well plates $\left(3 \times 10^{5}\right.$ cells per well). After overnight, adding Mito-DHA $5(0,3$ and $10 \mu \mathrm{M})$ or pretreated by $5 \mathrm{mM}$ NAC (Beyotime, China) and then cultured by Mito-DHA $5(0,3$ and $10 \mu \mathrm{M})$ for $12 \mathrm{~h}$, removed the culture medium, and then incubated with $10 \mu \mathrm{M}$ DCFH-DA at $37^{\circ} \mathrm{C}$ for $30 \mathrm{~min}$. After that, washing the cells three times with serum-free medium and then photographed by a fluorescence microscopy (DFC450C; Leica, Wetzlar, Germany).

\section{JC-1 assay}

MMP was detected through using $\mathrm{JC}^{-1}$ (Solarbio, Beijing, China) staining. In brief, T24 cells $\left(2.0 \times 10^{5}\right.$ cells per well) were plated in 12 -well plates. After $12 \mathrm{~h}$, treating cells with different concentrations $(0,3,10$ and $30 \mu \mathrm{M})$ of Mito-DHAs or DHA $(30 \mu \mathrm{M})$ for $48 \mathrm{~h}$. First of all, incubating with $\mathrm{JC}-1$ stain working solution $(0.5$ $\mathrm{mL})$ at $37^{\circ} \mathrm{C}$ for 20 minutes. Then washing the cells with $\mathrm{JC}-1$ staining buffer $(1 \times)$ for twice and resuspending the cells in $\mathrm{JC}^{-1}$ staining buffer $(1 \times)$. Fluorescence 
microscopy was used to measure MMP.

\section{Western blotting analysis}

Western blotting experiment was carried out with regular procedure. In brief, the protein bands incubated in first antibody for $15-18 \mathrm{~h}$ at $4^{\circ} \mathrm{C}$, washed them with PBST for 3 times (10 minutes per time), then incubated in secondary antibody for $1 \mathrm{~h}$ at room temperature. After this, washed 3 times again. After added Pierce Super Signal chemiluminescent substrate (Rockford 1L), imaging on Chemi Doc (Bio-Rad, USA).

\section{Data analysis}

We used the GraphPad Prism software to analyze our data and the mean \pm SD to present results. The statistical significance of the data is indicated as: ${ }^{*} p<0.05$, $* * \mathrm{p}<0.01$ and $* * * \mathrm{p}<0.001$. 


\section{Results}

\section{Mito-DHA5 inhibits the proliferation of T24 cancer cell line}

The anti-proliferation activity of Mito-DHA 5 and DHA in T24 cells were assessed by MTT assay. As shown in Fig. 1B, incubation of T24 cells with different concentrations of Mito-DHA 5 for $24 \mathrm{~h}, 48 \mathrm{~h}$ and $72 \mathrm{~h}$ resulted in reduction of the cell viability as compared with DHA in a dose-dependent manner and time-dependent manner. At the $3 \mu \mathrm{M}$, the cell viabilities of $\mathrm{T} 24$ were $87.94 \pm 1.97 \%$ for $24 \mathrm{~h}$, $54,40 \pm 1.47 \%$ for $48 \mathrm{~h}$ and $50.44 \pm 3.79 \%$ for $72 \mathrm{~h}$, respectively. At the $10 \mu \mathrm{M}$, the cell viabilities of T24 were $59.91 \pm 0.29 \%$ for $24 \mathrm{~h}, 30.02 \pm 0.43 \%$ for $48 \mathrm{~h}$ and $23.47 \pm 0.52 \%$ for $72 \mathrm{~h}$, respectively. At the $30 \mu \mathrm{M}$, the cell viabilities of $\mathrm{T} 24$ were $56.27 \pm 0.25 \%$ for $24 \mathrm{~h}, 23.95 \pm 0.83 \%$ for $48 \mathrm{~h}$ and $17.00 \pm 1.08 \%$ for $72 \mathrm{~h}$, respectively. At the $100 \mu \mathrm{M}$, the cell viabilities of T24 were $3.89 \pm 3.02 \%$ for $24 \mathrm{~h}, 3.12 \pm 1.69 \%$ for $48 \mathrm{~h}$ and $0.97 \pm 1.01$ for $72 \mathrm{~h}$, respectively. In order to evaluate the potency, half maximal inhibitory concentration $\left(\mathrm{IC}_{50}\right)$ value of $72 \mathrm{~h}$ for $\mathrm{T} 24$ cell lines were calculated for Mito-DHA $5(3.2 \pm 0.74 \mu \mathrm{M})$ and for DHA $(71.5 \pm 5.24 \mu \mathrm{M})$, respectively (Table 1). Mito-DHA 5 displayed 22 times higher inhibitory effects than DHA in the T24 cancer cell line.

Table 1. The anti-proliferation activity of Mito-DHA 5 and DHA against T24 cell line

\begin{tabular}{cc}
\hline Compounds & $\mathrm{IC}_{50}(\mu \mathrm{M})$ \\
\cline { 2 - 3 } & $\mathrm{T} 24$ \\
\hline Mito-DHA5 & $3.2 \pm 0.74$ \\
DHA & $71.5 \pm 5.24$ \\
\hline
\end{tabular}

\section{Mito-DHA 5 inhibits colony formation of T24 cells}

The effectiveness of Mito-DHA 5 to inhibit the clonogenicity of T24 cells was evaluated. The results showed that Mito-DHAs exerted potent inhibitory effects on T24 cells (Fig. 1C). At $4 \mu \mathrm{M}$ of Mito-DHA5, almost no colony formation was observed with T24 cells. The effect was dose-dependent and much more potent than the DHA treatment group at the same concentration. 
Fig. 1

A
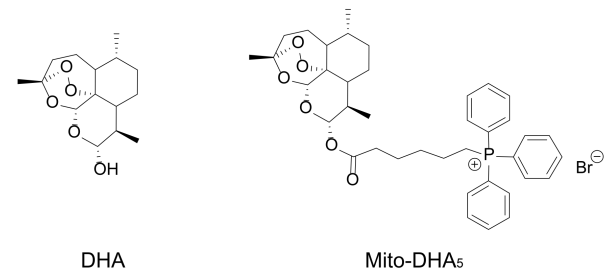

C

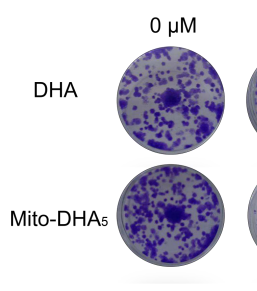

B

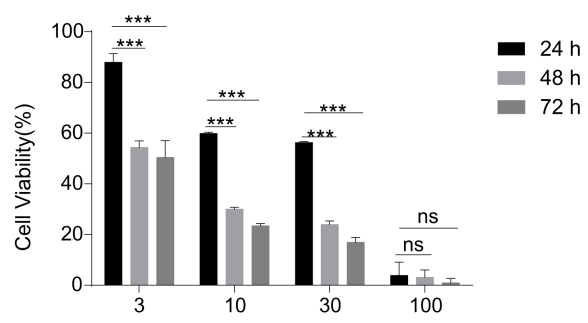

concentration $(\mu \mathrm{M})$

$\mathrm{D}$

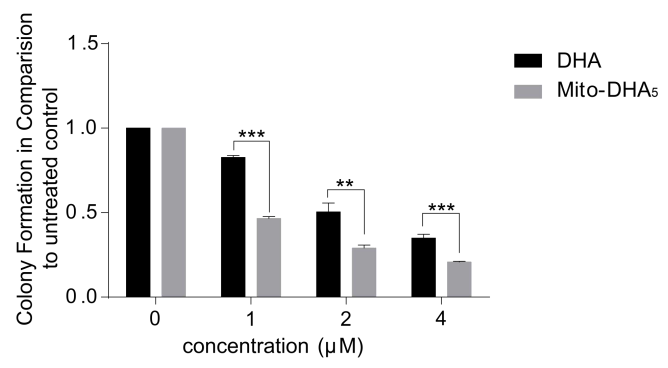

Proliferation inhibition of Mito-DHA 5 on bladder cancer T24 cells. (A). The structure of DHA and Mito-DHA5。(B). Treat T24 cells with 3, 10, 30, $100 \mu \mathrm{M}$ of Mito-DHAs for $24 \mathrm{~h}, 48 \mathrm{~h}$, and $72 \mathrm{~h}$, then observe the survival rate of the cells. (C). Evaluation of colony suppression by Mito-DHA5. T24 cells were treated for 7 days with Mito-DHA 5 and then stained with crystal violet to detect colony formation. (D). Quantification of the experiments conducted in panels. Wells were scanned at a wavelength of $550 \mathrm{~nm}$. Data are presented as mean \pm SD. $* \mathrm{P}<0.5,{ }^{*} \mathrm{P}<0.01, * * * \mathrm{P}<0.001$.

\section{Mito-DHA5 induces cell apoptosis in T24 cells}

We used annexin-V and PI double staining to determine whether the growth-inhibiting effects of Mito-DHA 5 in T24 cells was related to cell apoptosis. After the treatment of Mito-DHA 5 with different concentration for $48 \mathrm{~h}$, we analyzed the results by flow cytometry. As shown in Fig. 2A, Mito-DHA 5 induced significantly cell apoptosis in T24 cells in a dose-dependent manner. Apoptosis ratio at $30 \mu \mathrm{M}$ was 24.2\% (Fig. 2B). In order to explore whether the effect of Mito-DHA 5 in inducing cell apoptosis was stronger than DHA, the nuclei change in T24 was observed under a fluorescence microscope by Hoechst 33258. As shown in Fig. 2C, treatment with Mito-DHA $_{5}$ at $30 \mu \mathrm{M}$ induced more significant bright blue nuclei blebbing, nuclear rounding and shrinkage than treatment with DHA at $30 \mu \mathrm{M}$ in T24 cells. And the cell density of treatment with Mito-DHA 5 under the same concentration was much lower than that of treatment with DHA.

Mito-DHA5 decreases MMP in T24 cells 
To explore the effect of Mito-DHA 5 in MMP in T24 cells, we used the JC-1 staining method. After the treatment of Mito-DHA 5 with different concentration for $48 \mathrm{~h}$, the red fluorescence and green fluorescence were analyzed. An increase in green fluorescence intensity represents a decrease in MMP, while the red fluorescence intensity represents an increase in MMP. As shown in Fig. 2D and E, Mito-DHA 5 caused a decrease in MMP of bladder cancer T24 cells with a dose-dependent fashion. Compared with the ratio of green fluorescence/red fluorescence of DHA at $30 \mu \mathrm{M}$, Mito-DHA 5 increased the ratio at the same concentration, indicating the stronger effect of Mito-DHA 5 on decreasing capacity of MMP. 
Fig 2

A
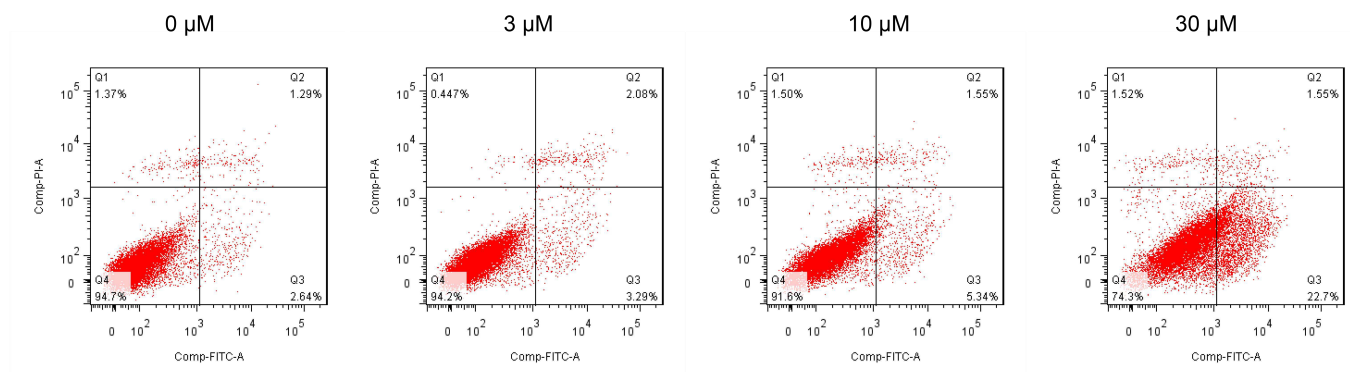

B

C
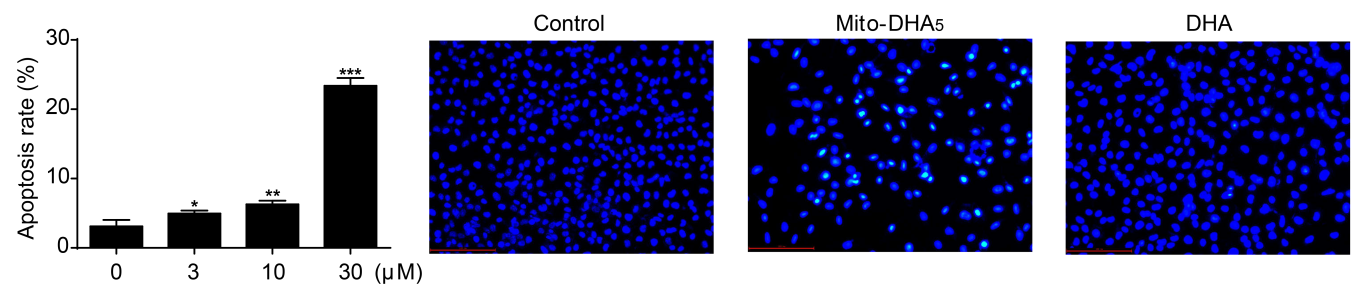

D
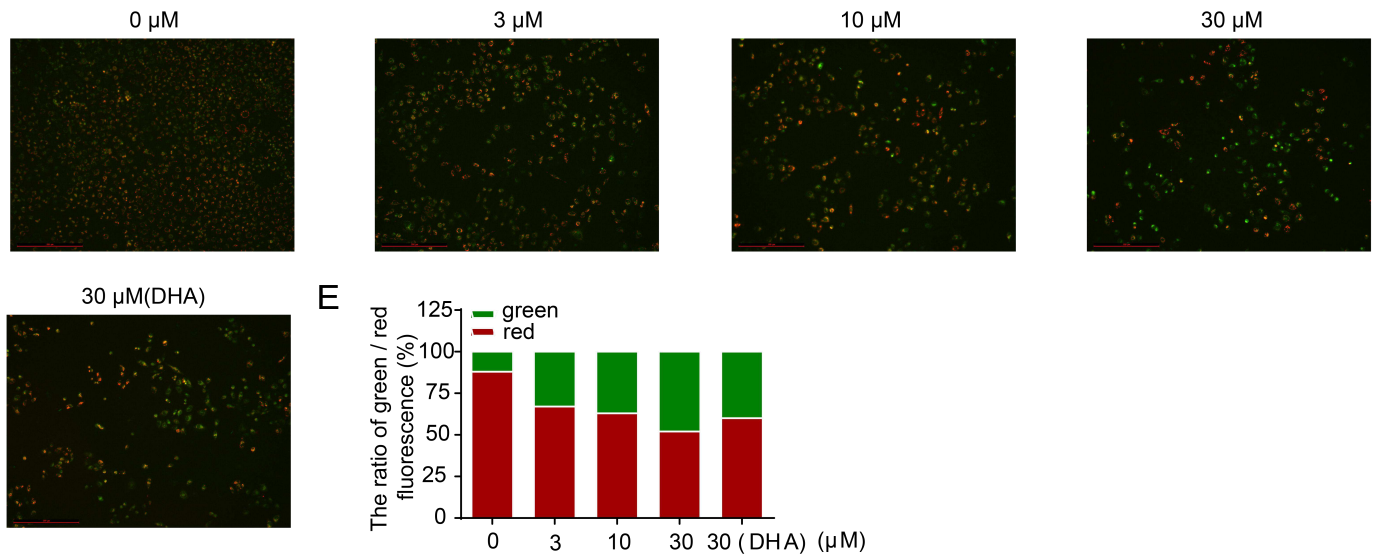

Effect of Mito-DHA 5 on cell apoptosis and MMP in T24 cells. (A). T24 cells were treated with Mito-DHA $5(0,3,10,30 \mu \mathrm{M})$ for $48 \mathrm{~h}$ and then assayed by flow cytometry analysis with Annexin V-FITC staining. (B) Quantification of apoptotic cells. (C). Treat T24 cells with $30 \mu \mathrm{M}$ of Mito-DHA 5 and DHA for $48 \mathrm{~h}$, respectively, stained with Hochest 33258, and viewed by fluorescence microscopy. Representative images were shown. Scale bar $100 \mu \mathrm{m}$. (D) T24 cells were treated with Mito-DHA $(3,10,30 \mu \mathrm{M})$ or DHA $(30 \mu \mathrm{M})$ for $48 \mathrm{~h}$ and then viewed by fluorescence microscopy after JC-1 staining. Increased green fluorescence represents a decrease in MMP. Scale bar $200 \mu \mathrm{m}$. (E). The ratio of green fluorescence to red fluorescence. Results are the mean $\pm \mathrm{SD}$ of three independent experiments. $* \mathrm{P}<0.5, * * \mathrm{P}<0.01, * * * \mathrm{P}<0.001$.

\section{Mito-DHA $A_{5}$ increases the ROS level in T24 cells}

We evaluated how treatment with Mito-DHA 5 in T24 cells influences the production of ROS tracked by DCFH-DA. Mito-DHA 5 induced an increase of ROS production in a dose-dependent manner and the pretreatment of NAC significantly 
reduced ROS production (Fig. 3A and B). The cytotoxicity of Mito-DHA 5 on T24 cells could be inhibited by NAC (Fig. 3C). The cell morphologic change was observed by fluorescence microscopy. The results showed that with the pretreatment of NAC, the induction of cell apoptosis was inhibited in T24 cells (Fig. 3D), indicating that Mito-DHA 5 induced T24 cell apoptosis dependent on ROS.

Fig. 3

A
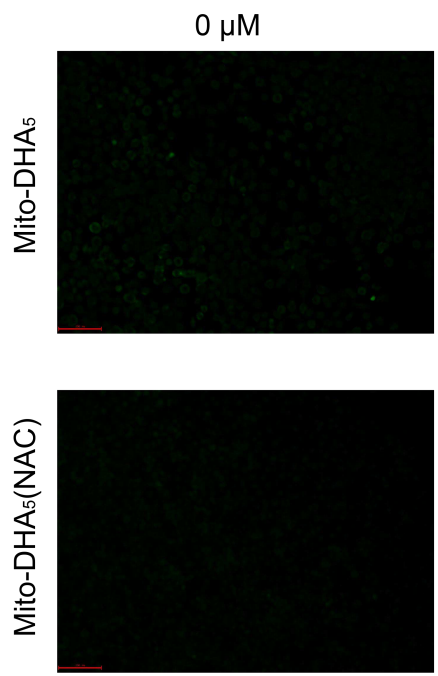

B

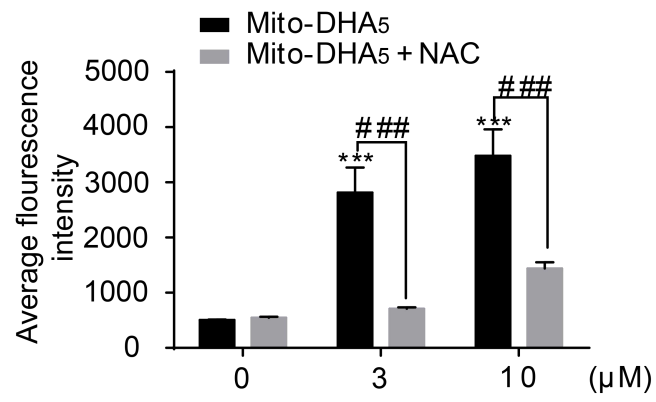

D

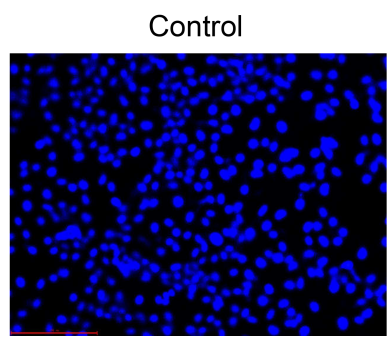

$3 \mu \mathrm{M}$
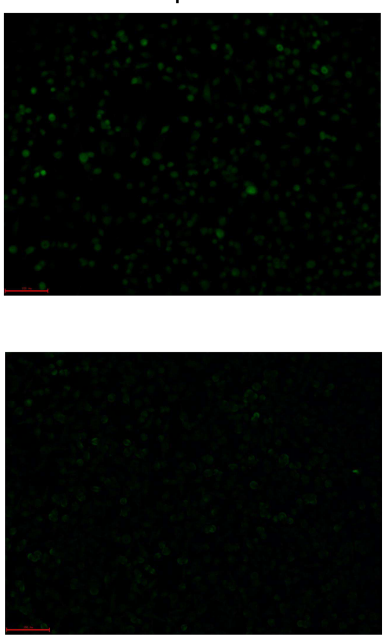

C
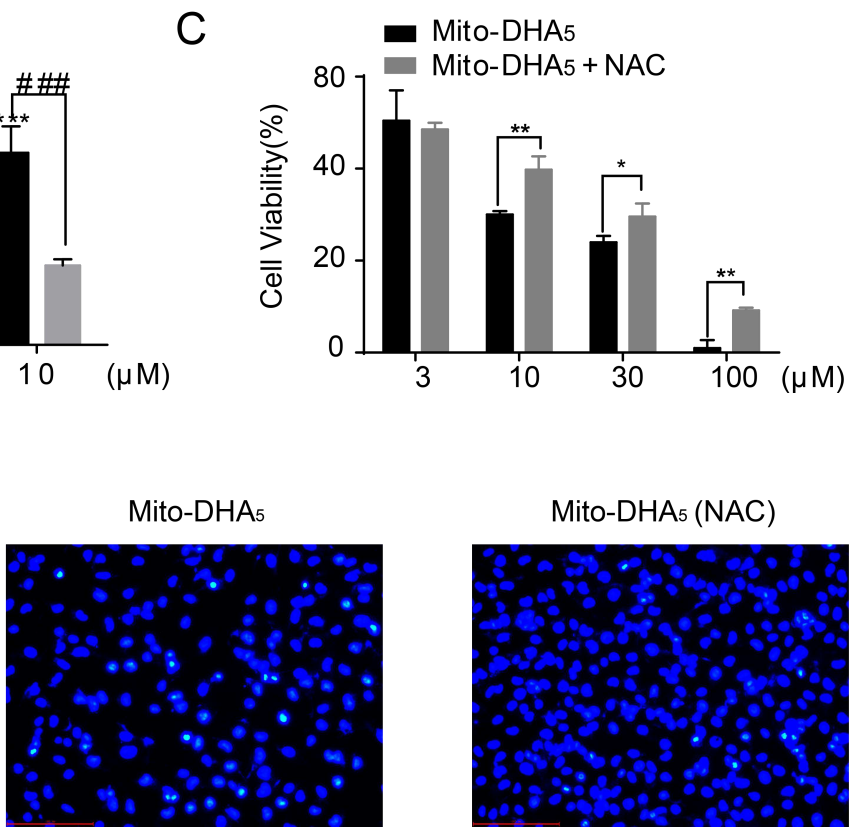

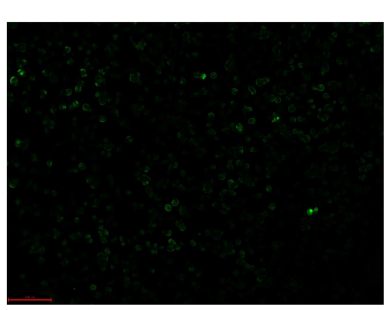

Mito-DHA5 (NAC)
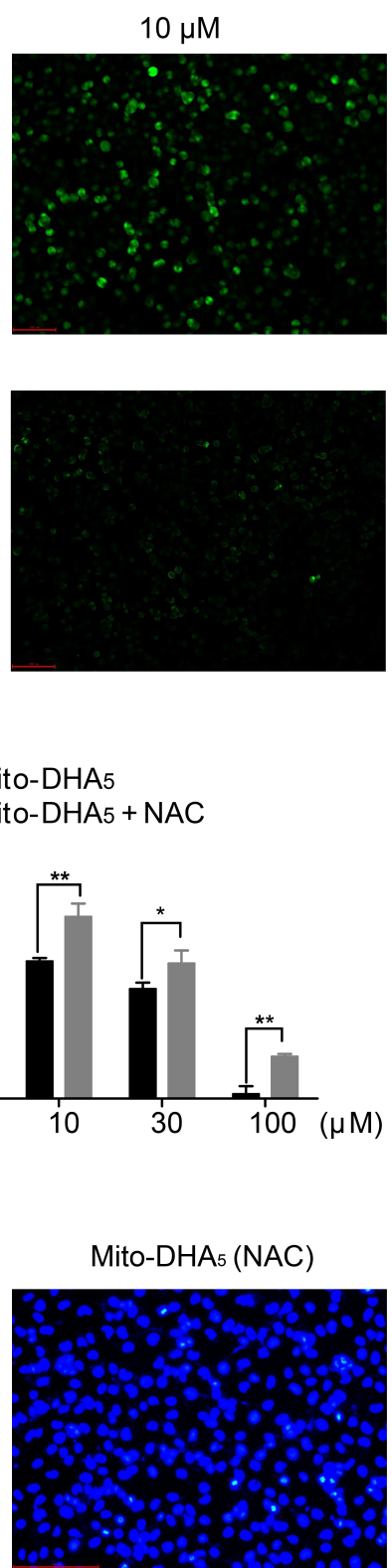

Effects of Mito-DHA 5 on ROS production. (A). T24 cells were treated with Mito-DHA 5 (0, 3, 10 $\mu \mathrm{M})$ in the presence or absence of pretreated with $5 \mathrm{mM} \mathrm{NAC}$ for $24 \mathrm{~h}$, then DCFH-DA $(10 \mu \mathrm{M})$ was loaded and cells were analyzed by fluorescence microscopy. Scale bar $100 \mu \mathrm{m}$. (B). Average 
fluorescence intensity of different treatment groups. (C). Effects of NAC on Mito-DHA5-induced cytotoxicity in T24 cells assessed by MTT. Cells were treated with Mito-DHA $5(3,10,30,100 \mu \mathrm{M})$ for $72 \mathrm{~h}$ in the presence or absence of pretreated with $5 \mathrm{mM}$ NAC. (D). T24 Cells were treated with Mito-DHA $5(10 \mu \mathrm{M})$ in the presence or absence of pretreated with $5 \mathrm{mM}$ NAC for $48 \mathrm{~h}$, stained with Hochest 33258, and viewed by fluorescence microscopy. Scale bar $100 \mu \mathrm{m}$. Data are presented as mean $\pm \mathrm{SD}$. ${ }^{*} \mathrm{P}<0.5,{ }^{* *} \mathrm{P}<0.01,{ }^{* * *} \mathrm{P}<0.001,{ }^{\#} \mathrm{P}<0.5,{ }^{\#} \mathrm{P}<0.01,{ }^{\# \#} \mathrm{P}<0.001$.

\section{Apoptosis effect of Mito-DHA5 was associated with mitochondrial pathway}

To further investigate whether Mito-DHA 5 influences the apoptosis-related protein expression (Fig. 4), we used Western blotting to detect the levels of mitochondrial pathway associated proteins. After the treatment of T24 cells with different concentration of Mito-DHA $5(0,1,3$ and $10 \mu \mathrm{M})$ for $24 \mathrm{~h}$, we found that Mito-DHA 5 treatment could down-regulate the expression of $\mathrm{Bcl}-2$ and up-regulate the expression of Bax in a dose-dependent manner. At the same time, Mito-DHA 5 activated caspase- 3 and increased the expression of cleaved caspase-3.

Fig. 4

A

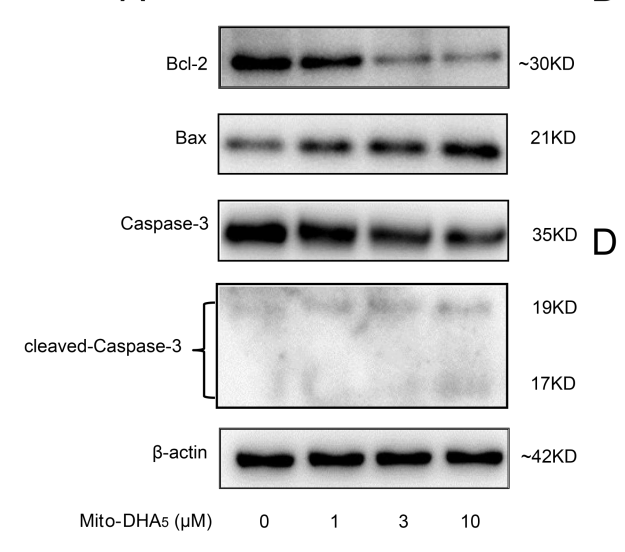

B
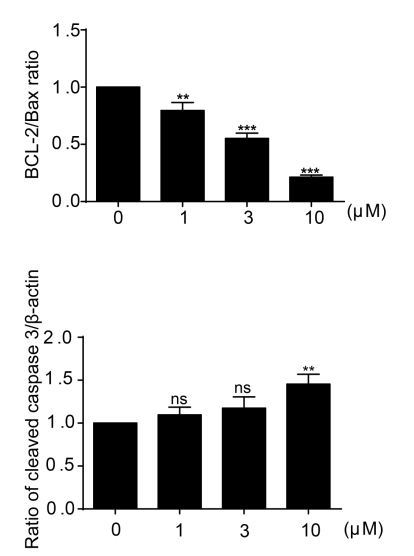

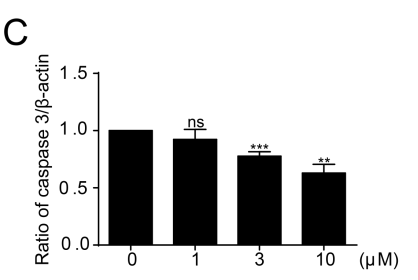

(A). The expression of cell apoptosis regulatory proteins Bcl-2, Bax and Caspase-3 in T24 cells after treatment Mito-DHA $5(0,1,3,10 \mu \mathrm{M})$. (B). The ratio of BCL-2/Bax protein expression. (C). The expression of Caspase-3 protein. (D). The expression of cleaved-Caspase3 protein. Data are the mean $\pm \mathrm{SD}$ of three independent experiments. ${ }^{*} \mathrm{P}<0.5,{ }^{* *} \mathrm{P}<0.01,{ }^{* * *} \mathrm{P}<0.001$. 


\section{Discussion}

Mito-DHA 5 is a newly synthesized mitochondrial-targeted artemisinin ester derivative. Our previous studies have found that this compound has great anti-tumor activity [12]. In this study, we have provided evidence that Mito-DHA 5 induced mitochondria-associated apoptosis, decreased mitochondrial membrane potential, increased ROS level, and leaded to caspase-3 activation associated with mitochondrial-dependent apoptosis pathway in T24 bladder cancer cells.

Apoptosis is a type of programmed cell death. This programmed cell death process is mediated by a variety of signal pathways (internal and external) triggered by a variety of factors (including cell stress, DNA damage, and immune monitoring) $[16,17]$. In this study, we evaluated the ability of Mito-DHA 5 to induce apoptosis of T24 bladder cancer cells. The ratio of apoptosis cells reached $24.2 \%$ after incubation with Mito-DHA 5 of $30 \mu \mathrm{M}$ in T24 cells. At the same concentration, Mito-DHA 5 had a stronger ability to induce cell apoptosis than DHA. Mitochondrial membrane potential is closely related to cell apoptosis, and the decrease of mitochondrial membrane potential indicates that cells may undergo early apoptosis [18]. We treated T24 cells with Mito-DHA 5 of different concentrations, and found that Mito-DHA 5 could significantly reduce the MMP at the concentration of $30 \mu \mathrm{M}$. In addition, the mitochondrial membrane potential of the DHA treatment group under the same concentration less than that of the Mito-DHA 5 treatment group. This result was consistent with the apoptosis result, and further told us that Mito-DHA 5 could induce apoptosis of T24 cells with much stronger apoptosis than that of DHA.

Reactive oxygen species (ROS) are a family of short-lived molecules and the production of ROS participates in radiotherapy by affecting downstream cell death signals $[19,20]$. Mitochondria, the place where cells breathe, are the main place where endogenous ROS is produced. A sharp increase in ROS in a short period of time will lead to the occurrence of cell apoptosis [21]. Our study showed that Mito-DHA 5 could cause an increase in the level of ROS in T24 cells, and this increase could be inhibited by the antioxidant NAC. At the same time, the killing effect of 
Mito-DHA 5 on T24 cells was weakened under the condition of NAC pretreatment. Besides, the results of fluorescence microscopy showed that the apoptosis ratio of the NAC pretreatment group decreased significantly under the same concentration of Mito-DHA 5 treatment. This implied us that Mito-DHA 5 -induced apoptosis might be related to the production of ROS.

The Bcl-2 family plays an important role in cell apoptosis [22]. The role of the Bcl-2 family in the regulation of apoptosis is generally described as anti-apoptotic and pro-apoptotic [23]. Bcl-2 is anti-apoptotic protein, while Bax is pro-apoptotic protein and the ratio of them is closely related to cell apoptosis. Mito-DHA5 treatment resulted in an obvious decrease in Bcl-2 protein expression and a significant increase in Bax protein expression in T24 cells. This indicated that Mito-DHA 5 inhibited the cell through the regulating of the expression of mitochondrial-mediated apoptosis-related protein Bcl-2/Bax. Caspase-3 is the downstream pathway of cell apoptosis and can be activated when cells is apoptosis [24]. Our research showed that after treating T24 cells with Mito-DHA, Caspase-3 can be activated and cleaved into 17 and $19 K D$ activators.

\section{Conclusions}

In conclusion, this research demonstrated that Mito-DHA 5 could induce apoptosis associated with mitochondrial-mediated pathway, decrease MMP, increased ROS levels and resulting in downregulation of the Bcl-2/Bax ratio and downstream activation the caspase-3. Collectively, these results suggest that Mito-DHA 5 holds promise for further development as a candidate for the treatment of bladder cancer. 


\section{Abbreviations}

DHA: Dihydroartemisinin; MMP: Mitochondrial membrane potential; ROS: Reactive oxygen species; DMSO: Dimethyl sulfoxide; DMEM: Dulbecco's modified Eagle's medium; MTT: 3-(4, 5-dimethylthiazol-2-yl)-2, 5- diphenyltetrazolium bromide

\section{Acknowledgments}

The helpful suggestions of anonymous referees and the Editor are acknowledged.

\section{Funding}

This work was supported by the Changsha Municipality Natural Science Foundation (No. kq2014081) to C. X; the open project of Hunan Normal University Medical College to L. M; and the National Natural Science Foundation of China (No. 81874212); Huxiang High-Level Talent Innovation Team (2018RS3072); Major Scientific and Technological Projects for Collaborative Prevention and Control of Birth Defect in Hunan Province (2019SK1012) and Key Grant of Research and Development in Hunan Province (2020DK2002) to X. Y.

\section{Author's contributions}

Linfan Xiao performed the experimental work and analyzed the data. Project administration and supervision were carried out by Xiaoping Yang, Cangcang Xu and Lingli Mu. Peiyu Lin helped to analyze the data. All authors contributed in writing the manuscript and approved it.

\section{Availability of data and materials}

All data and materials are contained and described within the manuscript.

\section{Ethics approval and consent to participate}

This article does not contain any studies with human or animal subjects performed by the authors.

\section{Competing interests}

The authors declare that they have no competing interests. 
Consent for publication

Not applicable. 


\section{References}

1. Richters A, Aben K, Kiemeney L: The global burden of urinary bladder cancer: an update. World Journal of Urology 2020, 38(6).

2. Siegel R, Miller K, Jemal A: Cancer statistics, 2019. CA: a cancer journal for clinicians 2019, 69(1):7-34.

3. Leow J, Bedke J, Chamie K, Collins J, Daneshmand S, Grivas P, Heidenreich A, Messing E, Royce T, Sankin A et al: SIU-ICUD consultation on bladder cancer: treatment of muscle-invasive bladder cancer. World journal of urology 2019, 37(1):61-83.

4. Rouprêt M, Babjuk M, Burger M, Capoun O, Cohen D, Compérat E, Cowan N, Dominguez-Escrig J, Gontero P, Hugh Mostafid A et al: European Association of Urology Guidelines on Upper Urinary Tract Urothelial Carcinoma: 2020 Update. European urology 2021, 79(1):62-79.

5. Koch G, Smelser W, Chang S: Side Effects of Intravesical BCG and Chemotherapy for Bladder Cancer: What They Are and How to Manage Them. Urology 2021, 149:11-20.

6. Grazzia N, Boaventura S, Garcia V, Gadelha F, Miguel D: Dihydroartemisinin, an active metabolite of artemisinin, interferes with Leishmania braziliensis mitochondrial bioenergetics and survival. Parasitology research 2021, 120(2):705-713.

7. Liu Y, Gao S, Zhu J, Zheng Y, Zhang H, Sun H: Dihydroartemisinin induces apoptosis and inhibits proliferation, migration, and invasion in epithelial ovarian cancer via inhibition of the hedgehog signaling pathway. Cancer medicine 2018, 7(11):5704-5715.

8. Yuan B, Liao F, Shi Z, Ren Y, Deng X, Yang T, Li D, Li R, Pu D, Wang Y et al: Dihydroartemisinin Inhibits the Proliferation, Colony Formation and Induces Ferroptosis of Lung Cancer Cells by Inhibiting PRIM2/SLC7A11 Axis. OncoTargets and therapy 2020, 13:10829-10840.

9. Jiang C, Li S, Li Y, Bai Y: Anticancer Effects of Dihydroartemisinin on Human Esophageal Cancer Cells In Vivo. Analytical cellular pathology (Amsterdam) 2018, 2018:8759745.

10. Paccez J, Duncan K, Sekar D, Correa R, Wang Y, Gu X, Bashin M, Chibale K, Libermann T, Zerbini L: Dihydroartemisinin inhibits prostate cancer via JARID2/miR-7/miR-34a-dependent downregulation of Axl. Oncogenesis 2019, 8(3):14.

11. Lu Z, Peng J, Zhang R, Wang F, Sun H, Fang Y, Wan D, Pan Z: Dihydroartemisinin inhibits colon cancer cell viability by inducing apoptosis through up-regulation of PPAR $\gamma$ expression. Saudi journal of biological sciences 2018, 25(2):372-376.

12. Xu C, Xiao L, Zhang X, Zhuang T, Yang X: Synthesis and biological activities of novel mitochondria-targeted artemisinin ester derivatives. Bioorganic \& Medicinal Chemistry Letters 2021, 39(55):127912.

13. Missiroli S, Perrone M, Genovese I, Pinton P, Giorgi C: Cancer metabolism and mitochondria: Finding novel mechanisms to fight tumours. EBioMedicine 2020, 59:102943.

14. Estaquier J, Vallette F, Vayssiere J, Mignotte B: The mitochondrial pathways of apoptosis. Advances in experimental medicine and biology 2012, 942:157-183.

15. Poupel F, Aghaei M, Movahedian A, Jafari S, Shahrestanaki M: Dihydroartemisinin Induces Apoptosis in Human Bladder Cancer Cell Lines Through Reactive Oxygen Species, Mitochondrial Membrane Potential, and Cytochrome C Pathway. International journal of preventive medicine 2017, 8:78. 
16. Ma A, Af A, Mf A, Al A, Al A, Ag A, Sz A, Ps B, Ci C, Mca D: Mitochondria as playmakers of apoptosis, autophagy and senescence. Seminars in Cell \& Developmental Biology 2020, 98:139-153.

17. Carneiro BA, El-Deiry WS: Targeting apoptosis in cancer therapy. Nature Reviews Clinical Oncology 2020, 17(Suppl. 13).

18. Zhang BB, Wang DG, Guo FF, Xuan C: Mitochondrial membrane potential and reactive oxygen species in cancer stem cells. Familial Cancer 2015, 14(1):19-23.

19. Quantitative proteomics reveals mitochondrial respiratory chain as a dominant target for carbon ion radiation: delayed reactive oxygen species generation caused DNA damage. Free radical biology \& medicine 2018.

20. Zulato E, Ciccarese F, Agnusdei V, Pinazza M, Nardo G, Iorio E, Curtarello M, Silic-Benussi M, Rossi E, Venturoli C: LKB1 loss is associated with glutathione deficiency under oxidative stress and sensitivity of cancer cells to cytotoxic drugs and $\gamma$-irradiation. Biochemical Pharmacology 2018, 156:479-490.

21. Mitochondria and Mitochondrial ROS in Cancer: Novel Targets for Anticancer Therapy. Journal of Cellular Physiology 2016:2570-2581.

22. Warren C, Wong-Brown MW, Bowden NA: BCL-2 family isoforms in apoptosis and cancer. Cell Death \& Disease 2019, 10(3).

23. Certo M, Moore V, Nishino M, Wei G, Korsmeyer S, Armstrong SA, Letai A: Mitochondria primed by death signals determine cellular addiction to antiapoptotic BCL-2 family members. Cancer Cell 2006, 9(5):351-365.

24. Jiang M, Qi L, Li L, Li Y: The caspase-3/GSDME signal pathway as a switch between apoptosis and pyroptosis in cancer. Cell Death Discovery. 